\title{
Heterogeneous integration of InGaAsP microdisk laser on a silicon platform using optofluidic assembly
}

\author{
Ming-Chun Tien • Aaron T. Ohta • Kyoungsik Yu • \\ Steven L. Neale • Ming C. Wu
}

Received: 11 September 2008 / Accepted: 16 December 2008 / Published online: 21 February 2009

(C) The Author(s) 2009. This article is published with open access at Springerlink.com

\begin{abstract}
Heterogeneous integration of InGaAsP microdisk lasers on a silicon platform is demonstrated experimentally using an optofluidic assembly technique. The 200-nm-thick, 5- and 10- $\mu$ m-diameter microdisk lasers are fabricated on InP and then released from the substrates. They are reassembled on a silicon platform using lateral-field optoelectronic tweezers (LOET). The assembled laser with 5- $\mu$ m diameter exhibits a threshold pump power of $340 \mu \mathrm{W}$ at room temperature under pulse condition. The heterogeneouslyintegrated InGaAsP-on-Si microdisk laser could provide the much needed optical source for CMOS-based silicon photonics. The small footprint and low power consumption make them attractive for optical interconnect applications. The optofluidic assembly technique enables efficient use of the III-V epitaxial materials in silicon photonic integrated circuits.
\end{abstract}

PACS $42.55 . \mathrm{Sa} \cdot 42.82 . \mathrm{Fv} \cdot 47.61 . \mathrm{Fg}$

M.-C. Tien $(\varangle) \cdot$ A.T. Ohta $\cdot$ K. Yu · S.L. Neale $\cdot$ M.C. Wu Department of Electrical Engineering and Computer Sciences, University of California, Berkeley, CA 94720, USA

e-mail: mctien@eecs.berkeley.edu

A.T. Ohta

e-mail: aohta@eecs.berkeley.edu

K. Yu

e-mail:ksyu@eecs.berkeley.edu

S.L. Neale

e-mail: sln2@eecs.berkeley.edu

M.C. Wu

e-mail:wu@eecs.berkeley.edu

Fax: +1-510-6436637

\section{Introduction}

Silicon photonics is an attractive technology for integrating electronic and photonic integrated circuits $[1,2]$. Nanoscopic photonic waveguides and optical modulators have been fabricated on silicon-on-insulator (SOI) substrates using complementary metal-oxide-semiconductor (CMOS) technologies [3, 4]. However, the progress for on-chip lasers that can be directly integrated with CMOS circuits has been much slower. Silicon Raman lasers have been demonstrated; but they still require external lasers for optical pumping $[5,6]$. Compound (III-V) semiconductor lasers have been grown directly on silicon substrate [7], but the growth temperature $\left(>400^{\circ} \mathrm{C}\right)$ is usually too high for post-CMOS processing. Direct bonding of III-V epitaxial materials on silicon offers a potential lowtemperature process that is compatible with post-CMOS fabrication. Oxygen plasma and the thermosetting polymer divinylsiloxane-benzocyclobutene (DVS-BCB) have been employed to bond III-V materials to Si $[8,9]$. AlGaInAsSi hybrid evanescent lasers [10] and InP-based microdisk lasers [11] have been demonstrated using such bonding techniques. However, integrating lasers on fully-processed CMOS wafers presents some challenges. In SOI-based silicon photonics, the silicon waveguides are buried underneath many (up to ten) layers of electrical interconnects, as illustrated in Fig. 1. Though bonding windows can be opened in the laser area, the nonplanar topography presents additional challenge for wafer bonding. An alternative approach has been proposed to add an "optical interconnect layer" on top of CMOS circuits. The optical interconnect layer, together with the heterogeneously-integrated lasers, is fabricated on separate SOIs and then bonded on top of the CMOS wafers [12]. The process, however, is more complicated. 


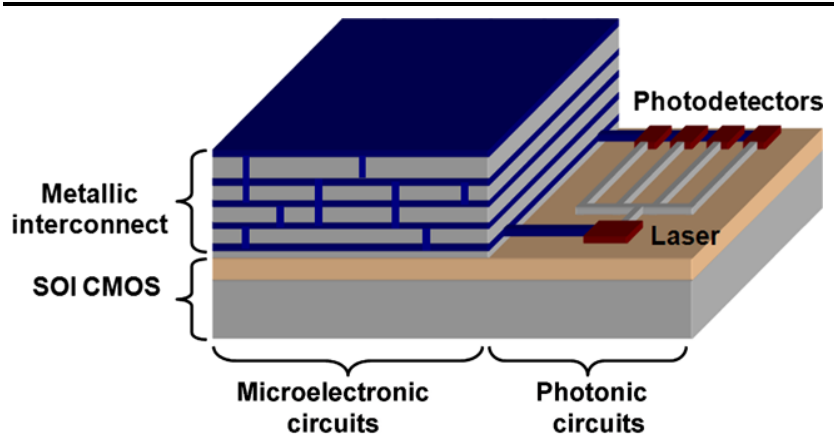

Fig. 1 Schematic illustrating the integration of III-V optoelectronic devices on SOI CMOS circuits

In this paper, we propose a novel optofluidic assembly technique to integrate prefabricated III-V semiconductor lasers on fully-processed CMOS wafers. The roomtemperature optofluidic assembly process is realized using lateral-field optoelectronic tweezers (LOET), which can overcome the topography issue. It also allows more efficient use of III-V epitaxial wafers. Using LOET, we have successfully assembled 200-nm-thick InGaAs/InGaAsP multiquantum well (MQW) microdisk lasers on a $\mathrm{Si}$ platform with alignment accuracy better than $0.25 \mu \mathrm{m}$. The transferred microdisk laser exhibits the same threshold pump power $(340 \mu \mathrm{W})$ as similar lasers on their native InP substrate.

\section{InP-based micordisk laser structure and fabrication}

The microdisk laser is fabricated using an InGaAs/InGaAsP/ InP MQW epitaxial wafer with a photoluminescence peak at $1550 \mathrm{~nm}$ [13]. Optical gain is provided by three 7-nmthick InGaAs quantum wells that are separated by $10-\mathrm{nm}$ thick InGaAsP (bandgap wavelength $\lambda_{g}=1.2 \mu \mathrm{m}$ ) barrier layers. The MQW layers are sandwiched between two symmetric InGaAsP optical confinement layers $\left(\lambda_{g}=1.1 \mu \mathrm{m}\right)$. The detailed epitaxial structure is shown in Table 1. The total thickness of the microdisk laser is intentionally kept very thin $(200 \mathrm{~nm})$ so that output light can be easily coupled evanescently to the $\mathrm{Si}$ waveguides. The microdisk lasers were grown by metalorganic chemical vapor deposition (MOCVD) on InP substrate.

Microdisk lasers with diameters of 5 and $10 \mu \mathrm{m}$ are fabricated using standard optical lithography and wet etching processes, as shown in Fig. 2. The circular microdisk pattern is transferred from photoresist to a silicon nitride hardmask by plasma etching. After removing the photoresist, the hardmask pattern is then transferred through the active layers to the InP sacrificial layer by a nonselective etchant of $0.5 \% \mathrm{Br}_{2}$ in methanol. Finally, after stripping the hardmask, the MQW microdisks are released by etching the InP sacrificial layer using a diluted hydrochloric acid $(\mathrm{HCl})$. The
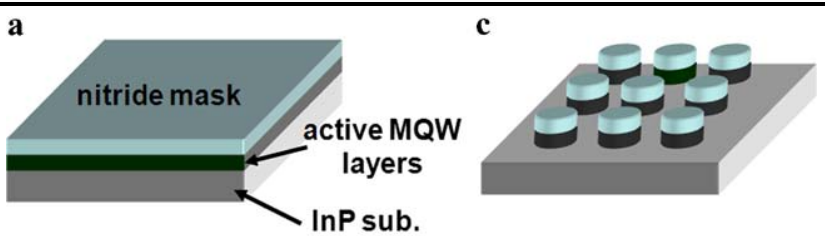

b
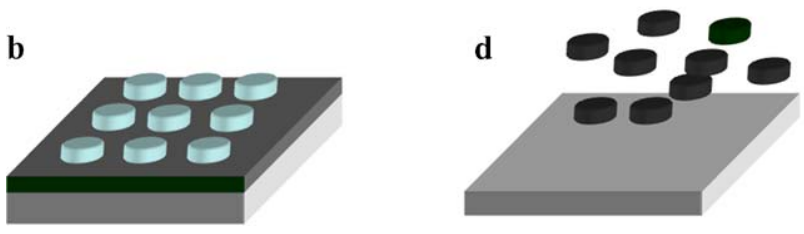

Fig. 2 Fabrication process of fully released InGaAs/InGaAsP multi-quantum-well (MQW) microdisk lasers. (a) Deposit silicon nitride hardmask. (b) Etch microdisk patterns in a silicon nitride hardmask. (c) Transfer the microdisk pattern from the hardmask to the active MQW layers using a nonselective etchant of $0.5 \% \mathrm{Br}_{2}$ in methanol. (d) Strip the nitride mask and release the microdisks by etching the InP sacrificial layer using diluted $\mathrm{HCl}$

microdisks are resuspended in ethanol for assembly onto a silicon platform. Figure 3 a shows the fabricated 5- $\mu \mathrm{m}$ diameter microdisks on a partially etched InP sacrificial layer. A close-up of the microdisk is shown in Fig. 3b, indicating a smooth sidewall for laser cavities with a low scattering loss.

\section{Lateral-field optoelectronic tweezers (LOET) for parallel assembly of microdisk lasers}

A post-CMOS parallel assembly technique that can seamlessly place and integrate multiple compound semiconductor lasers on desired locations is an attractive approach for fully integrated silicon photonics. Optoelectronic tweezers (OET) [14] is a massively parallel assembly technique that uses projected optical images to manipulate micro- and nanoscopic objects. It is a good candidate for post-CMOS assembly of semiconductor lasers. In this paper, lateral-field optoelectronic tweezers (LOET) [15], a variation of OET, is used to integrate InGaAsP microdisk lasers on a silicon platform. The LOET device is necessary in this process, as anisotropic objects such as the microdisks align with the electric field lines inside the LOET device. If the standard OET device were to be used, the microdisks would align with their major axis normal to the substrate.

The microdisk lasers support whispering gallery mode (WGM). Figure 4 shows the calculated magnetic field intensity profiles of the fundamental transverse electric (TE) WGM mode for 5- and 10- $\mu \mathrm{m}$-diameter microdisk lasers using the eigenmode expansion model. The electromagnetic field is concentrated near the edge of the microdisk. To minimize scattering loss and optical leakage, the diameters of the Si pedestals supporting the microdisk lasers are designed to 
Table 1 Epitaxial layer structure of microdisk lasers. The InGaAs multiple quantum wells with photoluminescence peak at $1550 \mathrm{~nm}$ are sandwiched by larger-bandgap optical confinement layers

\begin{tabular}{lll}
\hline Layer (bandgap) & Thickness & Description \\
\hline $1.1 \mu \mathrm{m}$-InGaAsP & $20 \mathrm{~nm}$ & Optical confinement layer \\
$1.2 \mu \mathrm{m}$-InGaAsP & $50 \mathrm{~nm}$ & Optical confinement layer \\
$1.2 \mu \mathrm{m}$-InGaAsP & $10 \mathrm{~nm} \times 4$ & $4-$ layer barriers \\
$\mathrm{In}_{0.53} \mathrm{Ga}_{0.47} \mathrm{As}$ & $7 \mathrm{~nm} \times 3$ & 3 -layer wells \\
$1.2 \mu \mathrm{m}$-InGaAsP & $50 \mathrm{~nm}$ & Optical confinement layer \\
$1.1 \mu \mathrm{m}-\mathrm{InGaAsP}$ & $20 \mathrm{~nm}$ & Optical confinement layer \\
InP & $350 \mu \mathrm{m}$ & Substrate (sacrificial layer) \\
\hline
\end{tabular}

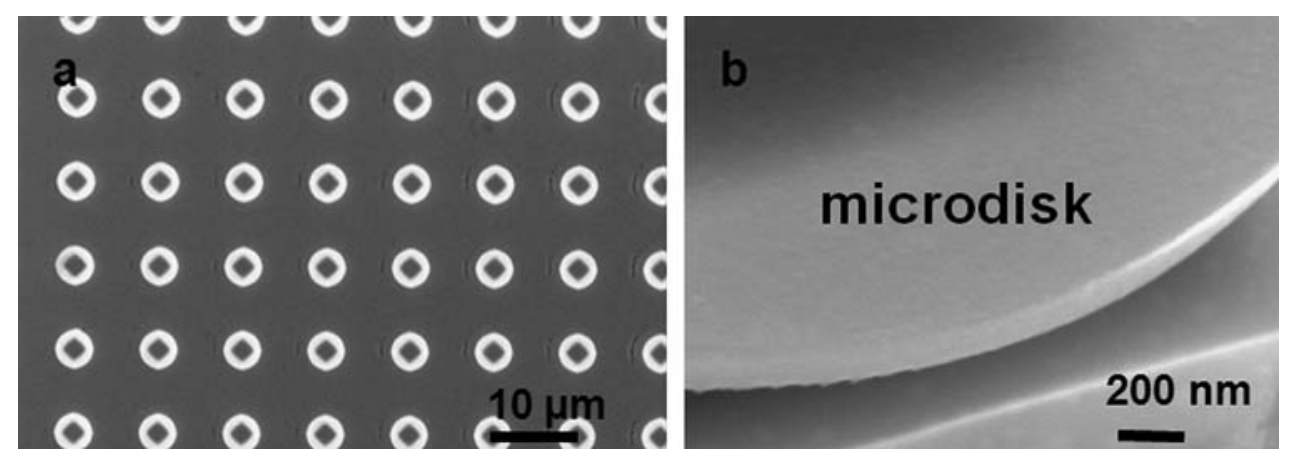

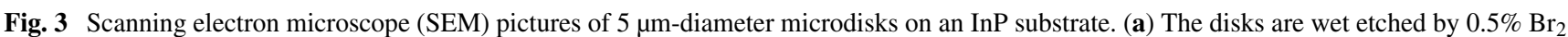
in methanol, followed by partial etch of the InP sacrificial layer using diluted $\mathrm{HCl}$. The rhombic grey region is an InP pedestal under the active InGaAsP/InGaAs multiple quantum wells. (b) A close-up image showing the disk sidewall after etching. The smooth sidewall provides a low loss cavity for the microdisk laser

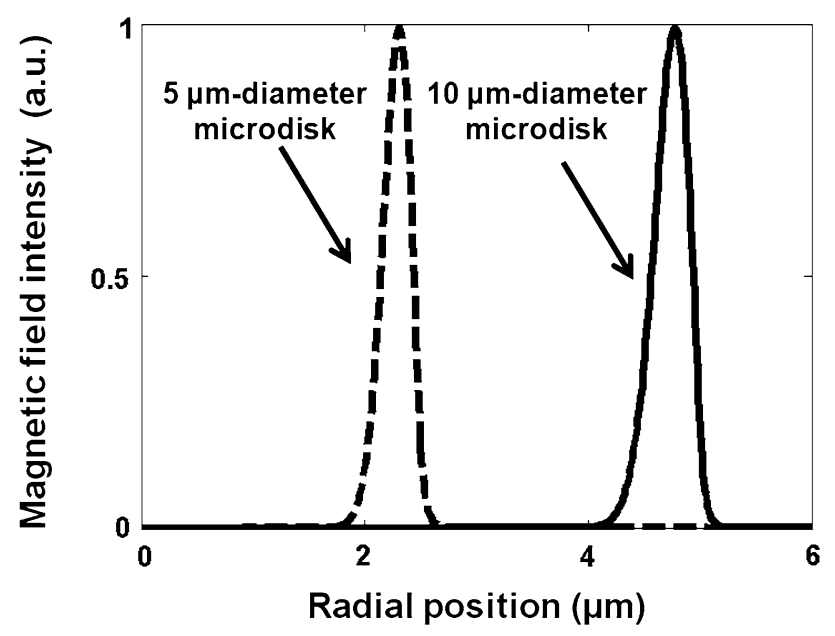

Fig. 4 The calculated magnetic field intensity profiles of the fundamental TE whispering gallery mode for 5 - and $10-\mu \mathrm{m}$-diameter microdisks using the eigenmode expansion model

be 3 and $7 \mu \mathrm{m}$, respectively, for the 5 - and $10-\mu \mathrm{m}$-diameter lasers [16].

The schematic of the LOET structure for assembling microdisk lasers is illustrated in Fig. 5a. A pair of photonconductive electrodes is patterned around a silicon pedestal, upon which the microdisk laser will be assembled. An ac voltage is applied across the electrodes, which consist of a 100 -nm-thick aluminum layer, topped by $0.8 \mu \mathrm{m}$ of amorphous silicon (a-Si). The a-Si layer functions as a lightcontrolled virtual electrode; the illuminated area switches from a low-conductivity state to a high-conductivity state. This causes the ac voltage drop to shift from the a-Si to the liquid between the a-Si electrodes. In other words, optical illumination creates a localized ac electric field in the liquid, whose gradient generates a dielectrophoretic (DEP) force $[14,15]$. The highest forces are near the edges of the LOET electrode (Fig. 6), which attract and align the microdisks in the center of the electrodes when the size of the disk is equal to or larger than the gap spacing.

The detailed fabrication process of the LOET structure is shown in Figs. 5b-5f. It is fabricated on an SOI wafer using a two-mask process. Silicon pedestals are first patterned in the top silicon layer by optical lithography and dry etching (Fig. 5c). A thin layer of oxide is grown by thermal oxidation to passivate the pedestals (Fig. 5d). The 100-nm-thick aluminum and 0.8- $\mu \mathrm{m}$-thick a-Si layers are then deposited and patterned to create the LOET electrodes (Figs. 5e, 5f). The pedestals are centered in the gap between the electrodes. At this point, the device is ready for the microdisk assembly 


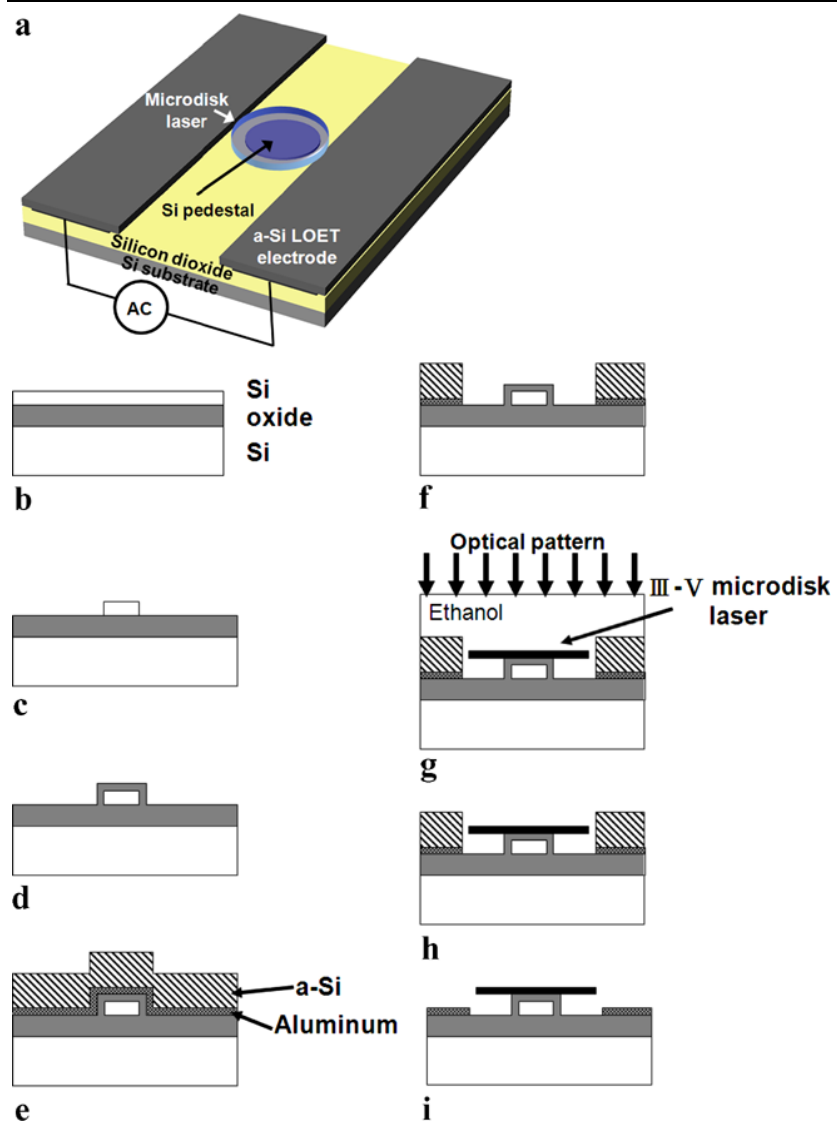

Fig. 5 (a) A schematic diagram of LOET with a microdisk laser assembled onto a pedestal. (b) The fabrication of pedestal-integrated LOET starts with an SOI wafer. (c) A Si pedestal is patterned using standard optical lithography. (d) A 50-nm-thick thermal oxide is grown to protect the pedestal from subsequent etches. (e) 100-nm-thick aluminum and $0.8-\mu \mathrm{m}$-thick a-Si are deposited by an e-beam evaporator and plasma-enhanced chemical vapor deposition (PECVD), respectively. (f) The LOET electrodes are defined by etching a-Si and aluminum. (g) The suspended microdisks are pipetted onto the substrate. The disks can then be trapped and positioned in solution. (h) The microdisks are held on the pedestals until the solution dries. (i) The a-Si layer is removed to avoid interference with the optical mode of the microdisk

process (Figs. 5g, 5h), which will be described in Sect. 4. After assembly, the a-Si layer is removed by $\mathrm{XeF}_{2}$ etching at $40^{\circ} \mathrm{C}$ (Fig. 5i).

\section{Assembly}

The fabricated InP-based microdisks, suspended in $~ 90 \%$ ethanol, are pipetted onto the substrate. The LOET electrodes create an optically-induced DEP force which attracts microdisks to illuminated areas, and the microdisks selfalign in the gap between the electrodes. An optical pattern controlled by a computer is projected onto the LOET device through an objective, as shown in Fig. 7. A CCD is used to monitor the relative locations of the projected pat-

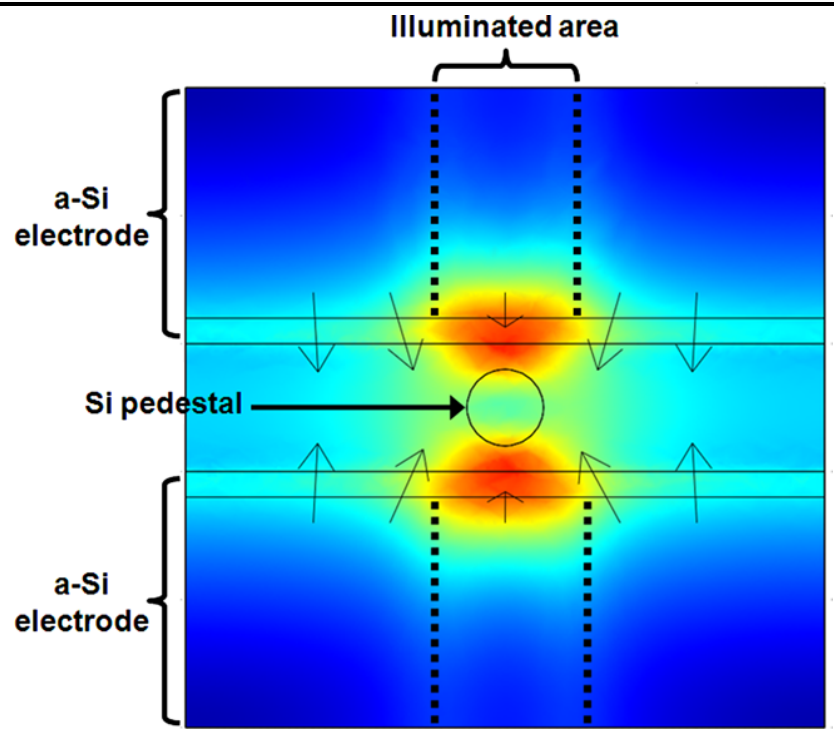

Fig. 6 Finite-element simulation of the electric field profile across the LOET electrodes. The arrows show the direction of the optically induced dielectrophoretic force. The strongest forces occur near the edges of illuminated electrodes



Fig. 7 Experimental setup for parallel assembly of microdisks on Si. A computer-controlled projector generates optical patterns to attract and transport microdisks to a designated position. A function generator is used to apply the bias voltage to the LOET device

tern, the microdisks, and the pedestals. The projector provides real-time control over the optical patterns, which allow transportation of the microdisks along the length of the LOET electrodes using an applied ac voltage of 1 to $10 \mathrm{Vpp}$ at $200 \mathrm{kHz}$. Once the disks are aligned over a pedestal, the applied voltage is increased to $20 \mathrm{Vpp}$ to hold the disks in place as the solution dries. Ethanol is used to minimize surface tension forces during drying, ensuring that the disks remain in place. After drying, the a-Si layer is removed to avoid interference with the optical modes in microdisks, as mentioned in Sect. 3. Figure 8 displays a series of microscope images showing the assembly process, as well as the scanning electron microscope (SEM) images of assembled microdisks with 5- (Fig. 8d) and 10- $\mu$ m diameters (Fig. 8h). As the gap between electrodes is $5 \mu \mathrm{m}$, the 5 - $\mu \mathrm{m}$-diameter 

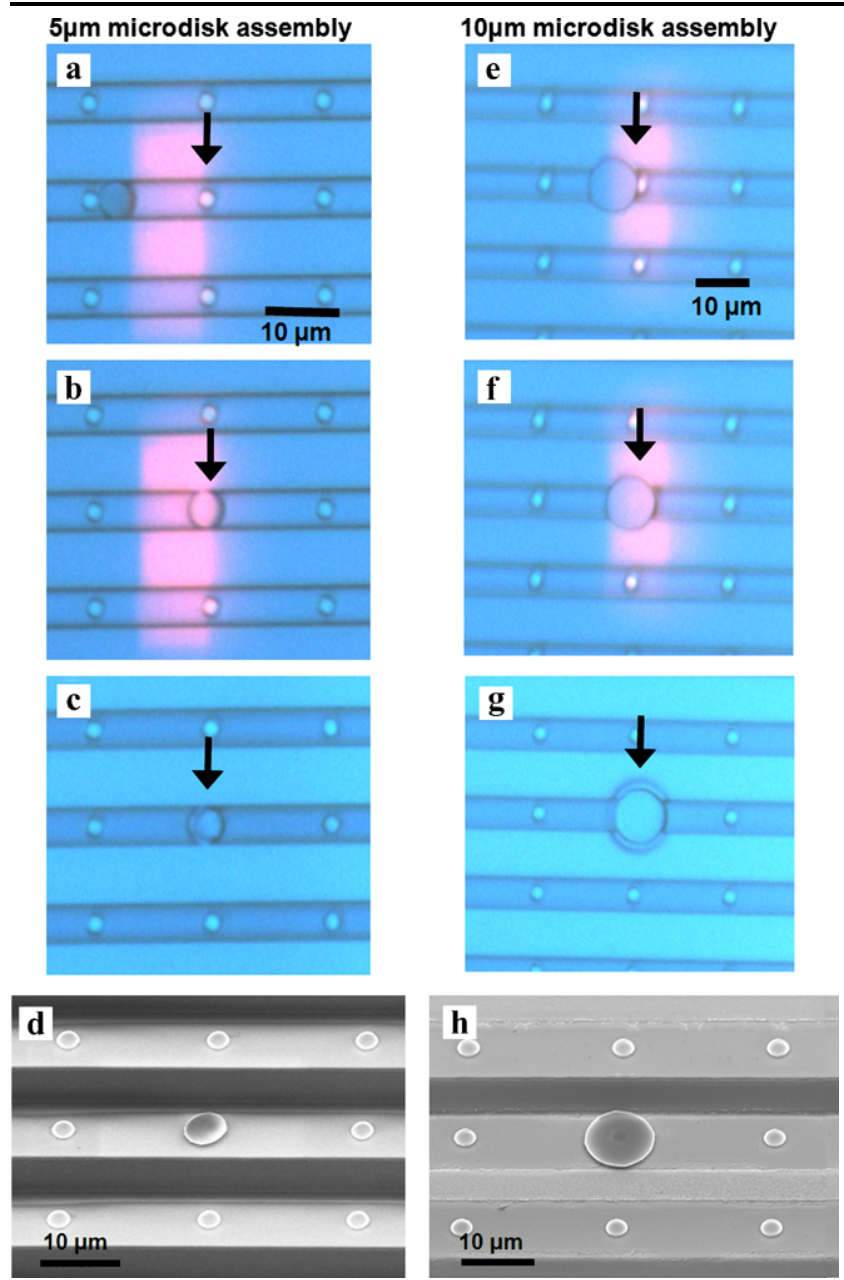

Fig. 8 Assembly of 5- $\mu$ m-diameter (a-d) and 10- $\mu$ m-diameter (e-h) InGaAs/InGaAsP microdisk lasers onto $3-\mu \mathrm{m}$-diameter silicon pedestals using lateral-field optoelectronic tweezers. (a, e) The initial positions of the microdisks. The optical trapping pattern, generated by a computer projector, is visible as a red rectangle. The microdisk is attracted towards the trapping pattern by optically induced dielectrophoretic force. The target $\mathrm{Si}$ pedestal for assembly is indicated by the arrow. (b, f) The microdisks are positioned over the target $\mathrm{Si}$ pedestal. The trapping force is then increased to immobilize the disks on the substrates. (c, g) The assembled microdisks remain in place after the liquid solution has dried. $(\mathbf{d}, \mathbf{h})$ SEM pictures of an assembled 5- and 10- $\mu \mathrm{m}$-diameter microdisks

microdisks fit between the electrodes (Figs. 8a-8d), while $10-\mu$-diameter microdisks self-align in the middle of the gap (Figs. 8e-8h). Both microdisks can be moved along the length of the electrodes by controlling the optical patterns.

The alignment accuracy of the assembled microdisks on the pedestals is also investigated [17]. As shown in Fig. 9a, the microdisk is self-aligned to the center of the gap in the horizontal direction (perpendicular to the electrodes) for those disks larger than the gap. The alignment along the electrodes is controlled by the projected light. The resulting alignment accuracy is measured in three LOET chips. The disk misalignment is $0.13 \mu \mathrm{m}$ in the horizontal direction and $0.25 \mu \mathrm{m}$ along the direction of the electrodes, as shown in Fig. 9 b.

\section{Optical measurement}

The assembled microdisk lasers are optically pumped at room temperature $\left(18^{\circ} \mathrm{C}\right)$ using a 780 -nm diode laser with $0.5-\mu$ s pulses and $20 \mathrm{kHz}$ repetition rate ( $1 \%$ duty cycle). The pump beam is focused onto the disk through a $40 \times$ objective, resulting in a beam spot size of $3 \mu \mathrm{m}$. The emitted light is collected by the same objective. An optical filter is used to block the pump light in the detection path. The filtered optical signal is coupled to a multimode fiber, and the output spectrum is measured by an optical spectrum analyzer.

The light-versus-pump curves of the microdisk lasers assembled on Si pedestals are shown in Fig. 10a. The threshold pump powers are 0.85 and $2.5 \mathrm{~mW}$ for the $5-$ and $10-\mu \mathrm{m}$ microdisk lasers, respectively. We estimate that $40 \%$ of the pump power is absorbed by the microdisks. The resulting effective thresholds are therefore 0.34 and $1 \mathrm{~mW}$, respectively. The light-vs-pump characteristics of unreleased microdisk lasers $(5-\mu \mathrm{m}$ diameter) on InP pedestals are also shown in Fig. 10a for comparison. There is no significant difference in the threshold pump powers between the unreleased and the assembled microdisk lasers, indicating that the microdisks are not damaged during the assembly process. The measured lasing spectra of the assembled lasers are shown in Fig. 10b. Both lasers exhibit single-mode operation. The peak lasing wavelengths are 1558.7 and $1586 \mathrm{~nm}$ for the 5- and $10-\mu \mathrm{m}$ microdisks, respectively.

Heating of the microdisk lasers eventually limits the maximum output power when the pump powers exceed 2 and $4 \mathrm{~mW}$ for the 5- and $10-\mu \mathrm{m}$ microdisks, respectively (Fig. 10a). The thermal resistances of the assembled lasers are higher than the unreleased lasers. The 3 - $\mu \mathrm{m}$-thick buried oxide under the Si pedestals substantially increases the thermal resistance due to the low thermal conductivity of silicon oxide $\left(\mathrm{SiO}_{2}\right)$. The poor contact between the microdisk lasers and the silicon pedestals also contributes to the high thermal resistance. Preliminary experiments have shown that a low temperature thermal annealing (at $300^{\circ} \mathrm{C}$ ) after assembly could improve bonding and increase the maximum output power.

\section{Conclusion}

We have demonstrated a new optofluidic technique for the parallel assembly of III-V microdisk lasers on silicon platforms. Using lateral optoelectronic tweezers (LOET), 
Fig. 9 (a) SEM image of an aligned microdisk on $\mathrm{Si}$ pedestal. (b) Alignment accuracy of assembled microdisks on three separate chips. The disk is self-aligned to the center of the gap in the horizontal direction due to symmetric DEP force. The vertical position is controlled by the projected light pattern

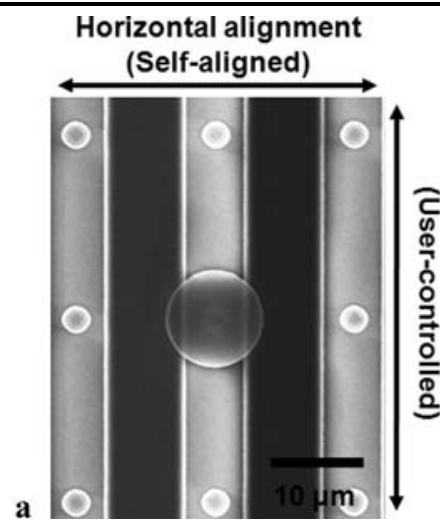

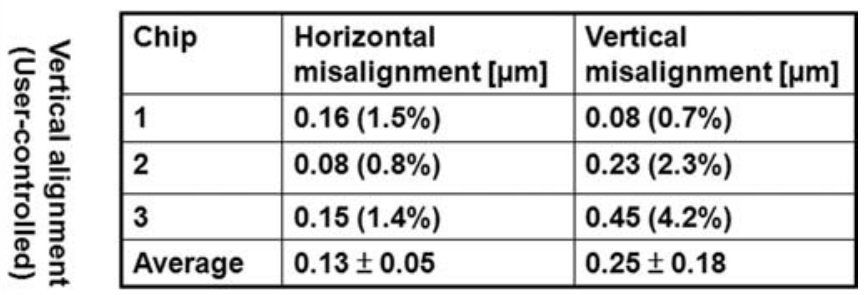

b

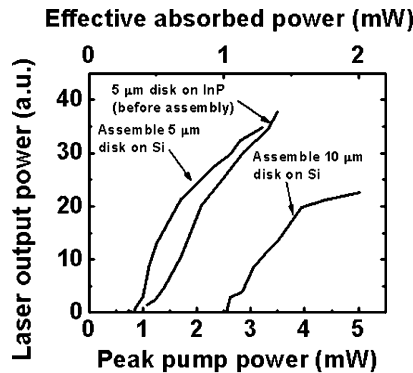

a

Fig. 10 (a) The light-vs-pump characteristics of the assembled microdisk lasers on $\mathrm{Si}$. About $40 \%$ of the pump power is absorbed by the microdisks. The effective threshold pump powers are therefore 0.34 and $1 \mathrm{~mW}$ for laser diameters of 5 and $10 \mu \mathrm{m}$, respectively. The data for a partially released laser ( $5 \mu \mathrm{m}$ diameter) on InP substrate is also shown for comparison. (b) Lasing spectra of assembled microdisks on $\mathrm{Si}$. Single mode operations are observed for both lasers

InGaAs/InGaAsP microdisk MQW lasers with diameters of 5 and $10 \mu \mathrm{m}$ are assembled on silicon pedestals with $<0.25 \mu \mathrm{m}$ alignment accuracy. The effective threshold pump power of the 5 - $\mu \mathrm{m}$-diameter laser is $0.34 \mathrm{~mW}$ at room temperature under pulsed mode excitation. We believe this parallel optofluidic assembly technique is particularly suitable for integrating compact III-V microdisk lasers on fully processed silicon CMOS electronic-photonic integrated circuits.

Acknowledgements The authors would like to thank the funding support of the DARPA University Photonic Research (UPR) program through the contract HR0011-04-1-0040 and the NSF Engineering Research Center for Integrated Access Networks (CIAN). The devices are fabricated at Berkeley Microfabrication Laboratory.

Open Access This article is distributed under the terms of the Creative Commons Attribution Noncommercial License which permits any noncommercial use, distribution, and reproduction in any medium, provided the original author(s) and source are credited.

\section{References}

1. D.A.B. Miller, Proc. IEEE 88, 728-749 (2000)

2. G. Chen, H. Chen, M. Haurylau, N.A. Nelson, D.H. Albonesi, P.M. Fauchet, E.G. Friedman, Integration the VLSI Journal 40, 434-446 (2007)

3. Y.A. Vlasov, S.J. McNab, Opt. Express 12, 1622-1631 (2004)

4. A. Liu, R. Jones, L. Liao, D. Samara-Rubio, D. Rubin, O. Cohen, R. Nicolaescu, M. Paniccla, Nature 427, 615-618 (2004)

5. O. Boyraz, B. Jalali, Opt. Express 12, 5269-5273 (2004)

6. H. Rong, R. Jones, A. Liu, O. Cohen, D. Hak, A. Fang, M. Paniccia, Nature 433, 725-728 (2005)

7. G. Balakrishnan, A. Jallipalli, P. Rotella, S.H. Huang, A. Khoshakhlagh, A. Amtout, S. Krishna, L.R. Dawson, D.L. Huffaker, IEEE J. Sel. Top. Quantum Electron. 12, 1636-1641 (2006)

8. D. Pasquariello, K. Hjort, IEEE J. Sel. Top. Quantum Electron. 8, 118-131 (2002)

9. G. Roelkens, J. Brouckaert, D. Van Thourhout, R. Baets, R. Notzel, M. Smit, J. Electrochem. Soc. 153, G1015-G1019 (2006)

10. A.W. Fang, H. Park, O. Cohen, R. Jones, M.J. Paniccia, J.E. Bowers, Opt. Express 14, 9203-9210 (2006)

11. J. Van Campenhout, P. Rojo-Romeo, P. Regreny, C. Seassal, D. Van Thourhout, S. Verstuyft, L. Di Cioccio, J.M. Fedeli, C. Lagahe, R. Baets, Opt. Express 15, 6744-6749 (2007)

12. H.T. Hattori, C. Seassal, E. Touraille, P. Rojo-Rmeo, X. Letartre, G. Hollinger, P. Viktorovitch, L. Di Cioccio, M. Zussy, L. El Melhaoui, J.M. Fedeli, IEEE Photonics Technol. Lett. 18, 223-225 (2006)

13. M.-C. Tien, A.T. Ohta, K. Yu, L.C. Chuang, A. Jamshidi, S.L. Neale, C. Hou, C. Chang-Hasnain, M.C. Wu, in Conference on Lasers and Electro-Optics (CLEO 2008), 2008

14. P.Y. Chiou, A.T. Ohta, M.C. Wu, Nature 436, 370-372 (2005)

15. A.T. Ohta, P.-Y. Chiou, H.L. Phan, S.W. Sherwood, J.M. Yang, A.N.K. Lau, H.-Y. Hsu, A. Jamshidi, M.C. Wu, IEEE J. Sel. Top. Quantum Electron. 13, 235-243 (2007)

16. M. Fujita, A. Sakai, T. Baba, IEEE J. Sel. Top. Quantum Electron. 5, 673-681 (1999)

17. A.T. Ohta, M.-C. Tien, K. Yu, S.L. Neale, M.C. Wu, in 2008, Digest of the IEEE/LEOS Summer Topical Meetings, 2008, pp. 207208 